\title{
5. Equipped for Case-Making
}

In this chapter, I trace the question of agency related to dispositifs. I take up the crucial insight of material-semiotic approaches that practices of governing are enabled and mediated by material-discursive arrangements (Latour 2010; Scheffer 2010, 45) of government. Caseworkers are becoming material-discursively assembled or equipped to be able to assemble cases and enact the dispositif (see Rabinow 2003). ${ }^{1}$

In the first subchapter (5.1), I assemble individual and collective agentic formations that are crucial for enacting the dispositif in practices of case-making: primarily caseworkers, sections and 'the office'. I suggest some associations and socio-material technologies to be central for assembling them, namely associations that compose a proxy of the 'nation', membership devices, ritualised events of assembling collectives and associations of super-vision. In the second subchapter (5.2), I introduce central devices for re-cording lives in terms of asylum: material-discursive tools for acting upon the lives of those who claim asylum in prosaic practices of case-making. I distinguish between various types of devices, namely recording, inscription, coordinating, and writing devices. Together, these devices are crucial mediators for assembling asylum cases towards their resolution (see Part II).

1 This also has consequences for the crucial issue of accountability (see also section 8.1.4): viewing practices as mediated by such composite agentic formations shifts what accountability means by both altering what counts or matters (increasingly numbers), and who accounts (increasingly nonhuman mediators). 


\subsection{Assembling Agentic Formations}

From now on, when we speak of actor we should always add the large network of attachments making it act. As to emancipation, it does not mean "freed from bonds" but well-attached. (Latour 2005, 217-18)

In this subchapter, I address the following question: What does it take to assemble an administration - as a composite reality, a collective - and administrators - as individuated parts of this collective? A few things, I suggest, have to be achieved: first, one needs to be able to distinguish those who belong to the collective(s) from those who do not. Second, one needs to enrol people and things in the manufacturing of decisions* and the enactment of the dispositif. And third, who and what is enrolled needs to be connected through differentiated webs of 'super-vision'. This account intends to destabilise the prevailing view of public administrations of something out there, already assembled, naturally operating, administering whatever task they are entrusted with.

\subsubsection{Caseworkers: Lone Warriors?}

That's quite interesting with the new one [caseworker], who just started. He has his office next to mine. On the one hand, I almost feel a bit sorry for him, because he's now also in the situation in which you are [he is] a little overstrained. You know, that's totally normal ... But it's really cool to watch what you really go through. (Interview with caseworker, reception centre, autumn 2013)

New caseworkers (in German, Fachspezialistinnen Asyl) start more or less from scratch. As the quotation above indicates, they are usually thrown in at the deep end: although they attend the basic training for new caseworkers, they usually have to start doing casework as soon as their jobs start. Most of the caseworkers I met in the basic training shared both the feelings of excitement about all the new things they learned and of being overstrained, as the caseworker observed with her co-worker in the quote above (and remembered she also went through). Caseworkers are thus connected through the shared experiences of learning and doing casework. Early in my fieldwork, I was struck by the paradoxical state caseworkers appeared to have: as composite "actants" (Latour, 2005) constituted by institutional associations, and 
yet strangely dissociated in their everyday work on parts of cases as sort of "lone warriors" (Interview with caseworker, 2013). Both states of caseworkers, I suggest, are essential for understanding the governing of asylum. I will thus briefly trace some of the central associations as well as some of the dissociations that constitute caseworkers.

Caseworkers are at the heart of the asylum procedure: they process cases in their various stages in the reception centres and headquarters of the asylum office. Caseworkers in the Swiss asylum office have different professional and educational biographies - a colourful mix of social scientists and jurists, linguists, historians and a veterinarian. Some of those entering public administration as caseworkers already have work experience in the field of migration or asylum, ${ }^{2}$ while others have no previous experience. Asylum casework broadly consists of two core components: conducting interviews with asylum claimants and writing legally binding decisions. According to senior officials who are involved in the hiring of new caseworkers, different competences are required for casework: socio-cultural communication and interview skills, legal skills, and linguistic skills. Depending on the relative emphasis of these competences, more or fewer jurists, social scientists, or linguists were recruited during certain periods (for an extensive discussion of the recruiting process and the 'types' of people hired in the asylum office see (Affolter 2017, 23-27; Miaz 2017, 185-92). Generally, a balanced mix of different backgrounds seems to be valued by most members of the office. In the basic training, I was in good company of social scientists, linguists, philosophers, and jurists.

In the basic training for new caseworkers, their work was characterised and framed in a certain way. One of these framings appears particularly meaningful for understanding how caseworkers are associated with 'the state': "In your work, you will perform legal acts in the name of Switzerland" (Fieldnotes, basic training for new caseworkers, autumn 2012). This statement resonates with the idea that the 'state' takes form where acts are performed in its name (see also Gupta 1995; Reeves 2013). By explicitly referring to "Switzerland", the instructor moreover invoked the idea of the nation-

\footnotetext{
2 I met a few people who had worked as relief organisation representatives in asylum hearings before becoming caseworkers. And some employees of the Federal Administrative Court 'changed sides' to the asylum office when the court was moved from Bern to (faraway) St. Gallen in mid-2012.
} 
state (see Abrams 1988) and established a relationship of loyalty towards this transcendent figure: caseworkers enact the asylum dispositifas proxies of the Swiss nation-state. The framing of acts as "legal", moreover, underlines caseworkers' role as being rooted in a notion of the rule of law. This appears crucial for the foundational legitimacy of resolving questions of "life and death" in an institution (see Douglas 1986, 111). Such a resolution is at stake in the governing of asylum, which means - according to a self-declaration in the training - that "we decide about who is granted protection in our country and who does not require it" (Fieldnotes, basic training for new caseworkers, autumn 2012).

This second framing of what the work of caseworkers 'is all about', I think, is characteristic of how this essential delegation is performed: through the collectives that are invoked. A first 'we' - the asylum office - is enacted, a collective who decides "who is granted protection". It thus never remains on the shoulders of an individual person to decide, but rather on a caseworker who is part of the office collective (see also subchapter 8.1). A second 'we' is invoked: the nation-state where protection is sought. While, according to the first quote above, caseworkers solely act in the name of this second 'we' (Switzerland), the framing "in our country" in the second quote implies that they are a part of this more comprehensive 'we'. And finally, a third collective is performed: that of the subjects of asylum ("who is granted protection ... and who does not require it"). ${ }^{3}$ Thus, to become caseworkers, humans entering the dispositif become associated with multiple collectives: an organisational one, the asylum office with the competence of "deciding" on asylum applications, and a national one, Switzerland, in whose name it is to decide. As caseworkers, they are delegated the ethical quandary of selecting, according to legal standards, who is eligible to reside "in our country" (see also Fassin 2013) from the collective of 'applicant others'.

Caseworkers can thus be considered composite actants as they are unthinkable outside the collectives that confer the authority to assess eligibility to them. But, paradoxically, they also portray themselves as 'lone

3 These are in fact two questions. Why not "who is granted protection and who is not"? The second part can be read as an explication of "who is not granted protection", namely "who does not require it". But it can also be read as an implicit reference to the moral weight of the question to be answered by the institution: the necessity to protect (or the deservingness of) someone who is asking for protection (i.e., membership in the larger 'we'). 
warriors' in their everyday work. I also had the impression that case-making happens dissociated from others: case-making usually means a particular case file was assigned to a caseworker for the task at hand. Consequently, a lot of work takes place at the desk in the office where caseworkers assemble some types of records on their computer by filling forms or writing standard letters, for instance. In the hearings that caseworkers conduct, they are not literally alone, but are nevertheless 'lone warriors' as 'representatives of the state'. When it comes to numbers - the crucial measure of administrative efficiency - every caseworker (and also every head of section) is individually responsible for the output she or he produces (see sections 8.2.2-3). Of course, meetings, training sessions and informal exchange with other caseworkers and seniors are crucial for the everyday life of a caseworker as well (see sections 5.1.3-4). Yet, actual casework typically implicates isolation from others until the records necessary for the case to be passed on or resolved are assembled (see subchapter 6.3). It seems important to keep this double constitution of caseworkers through both strong associations and dissociations in mind when both looking at the pragmatics of case-making.

\subsubsection{Membership Devices: Access and Insignia}

Becoming agentic as a caseworker is accomplished not only through being exposed to ways of organisational knowing (see Chapter 4) but is crucially mediated through what I call "membership devices". Membership devices enrol humans in the collectives of case-making and bestow them with agentic 'potential' and legitimacy. They are thus crucial for caseworkers' agentic formation. Such mundane devices mediate membership by providing access to the built and digital landscapes of the administration; by allowing identification as an official with a certain position in (a specific subdivision of) the administration; and by enabling the incorporation of individuals and various subdivisions of the collective into the administrative circuits of information and correspondence. Crucial about caseworkers' access to various spaces of case-making and their location inside them is: the enrolment of humans in governing asylum not only implicates that they become assembled to have a positionality to know and see asylum from but also becoming situated in a spatiotemporal location from which to enact the dispositif. 
Asylum case-making involves clearly delimited spacings and timings (see Gill 2009). ${ }^{4}$ All the entrance doors of the buildings in the headquarters require a badge; those in the reception centre in which I did fieldwork required a programmable key. Non-members of the administration have to register for a visitor card at one of the main gates. This was also my fate at the very beginning of my visits to the asylum office. With the visitor card, which I had to wear visibly on my clothes, I was clearly identifiable as a non-member. Already in the reception centre, access to and movement in the office wing was unobstructed, since I was provided with a key. For the last part of my fieldwork in the headquarters, I received a badge. For my bodily circulation in the asylum office and my feeling of belonging, both key and badge were significant. Without them I would have depended heavily on officials opening doors for me all day long. For 'real' officials, badges have another crucial function: mounted just behind the gates inside all the office buildings are devices that log staff work-time, which have to be touched with the badges when entering or leaving the building to register working hours. They render having the capacity to 'act as a bureaucrat' not only a matter of office space but also office time, a "chronotope" in Bakhtin's terms (Valverde 2014). Various transgressions of this office timespace occur - as in telework and evening and weekend shifts - but require special permissions and also foster contestation.

For my internship and fieldwork in the headquarters, I moreover received a "smartcard". ${ }^{5}$ The card is crucial to access the digital spaces of case-making: to log into a computer, to enter key databases, and write in the name of the state (see section 5.2.4). In short, it enables one to act as an official in very basic ways. The smartcard is thus a crucial device for a caseworker's agentic formation. This was also true for me: for the last part of fieldwork (as an intern), I not only received a smartcard but also a federal laptop, which I could plug in at every vacant workstation in the asylum office.

Once, access to my computer was not possible so I had to call the Federal Office of Information Technology, Systems and Telecommunication, inter-

4 As Cill (2009) has pointed out in the case of UK asylum sector workers, such spacings and timings that he considers a form of "presentational state power" come with particular ethical implications for encountering claimants.

5 I was in turn bound to the obligations of every caseworker concerning data and personal protection, information security, and the duty of confidentiality through a declaration of commitment. 
nally only referred to in the German acronym BIT (whose logo with the phone number was part of the standard blue desktop image of all computers in the office). After explaining my situation and providing my authentication, I was given a temporary substitute login. The authentication involved giving the computer scientist the answers to questions I had once named when I had received my smartcard. I smilingly gave the answers to the questions regarding the name of our first dog and my dream job as a kid, and jokingly added that he would probably learn a lot about federal employees by asking these questions. He laughed and confirmed my presumption. He said that they were sometimes quite intimate, and that he thinks many people do not expect to be asked these questions on the phone one day. ${ }^{6}$ This short episode certainly hints at the association of the asylum dispositif with another large assembly: that of federal IT. It moreover indicates the personalisation accompanying membership in the federal administration - including potentially confessionary encounters. In brief, keys and badge appear to mediate the corporal access to the built landscape of the administration, while the smartcard is crucial for accessing the digital infrastructure. But how is one identified as an official in the first place, and as one with a certain position in the administration?

Personal identifiers are key for the circulation of objects and registration of work on- and offline: every official receives a unique acronym, usually composed of three letters of the first and surname. My personal identifier, for instance, was the easily pronounceable "Poe". Such acronyms are omnipresent in the asylum office: they are used instead of or in addition to full names on records, sticky notes, in letter headers, meeting protocols, and various other forms of documents. Soon I knew the acronyms of all my co-workers. Some long-term employees I met markedly identified with their acronyms and people referred to them by their acronyms in their absence. In one case, people even mainly addressed a person by her or his acronym.

With the addition of the section identifier, the acronym is used to designate the case file location, to deliver case files to the right person in the office,

\footnotetext{
6 Such personal authentication questions evidently require a certain degree of intimacy, because the answer to them should not be found in your CV or elsewhere on the internet. But to draw a picture of the federal administration with information from this BIT database would be both a tempting and frightening possibility.
} 
but also represents an official in the digital databases (e.g. "1AV6 Poe"). ${ }^{7}$ This identifier that all officials have - together with the necessary interfaces and letterbox - associates them with the digital and analogue channels of circulation; it locates them inside the administration and it renders them addressable and their activities traceable in documents, folders, and databases.

Many more such devices are involved in assembling "communities of practice" (Wenger 2003). Another mundane but vital medium of membership is, for instance, the email client. Issuing emails with official state mail signatures made me aware of being located within the asylum community of practice - any recipient could use either the digital or the postal address to reach me as an 'official'. Certainly, both identifiers and email clients are mundane and self-evident elements of various types of office infrastructures today. Nevertheless, they play a widely neglected but important role in the mediation of membership and authorship as exclusive infrastructural devices. They also operate as both collectivising and individualising mediators - involved in the shaping of an 'organisation' and an 'official' (and all the subdivisions in between). Both do not just exist, they have to be actively composed: my argument here draws on Latour (2005) and what he called "individualisers or plugins":

to obtain 'complete' human actors, you have to compose them out of many successive layers, each of which is empirically distinct from the next. Being a fully competent actor now comes in discreet pellets or, to borrow from cyberspace, patches and applets, whose precise origin can be 'Coogled' before they are downloaded and saved one by one. (Latour 2005, 207)

Hence, officials as human actors are conceptualised to consist of more than mind and body, to be "a kind of machination, a hybrid of flesh, artifact, knowledge, passion, and technique" (N. Rose 1996, 38). They become composed through mental and bodily, analogous and digital technological trivialia, which appear as natural inhabitants of office spaces but are also amongst the plugins for their composition. But besides operating as individualisers, they also work as collectivisers - because they are shared, indicate membership and allow for authorship and thus the dispositif's enactment.

7 The first part of this longer identifier indicates the division (AV stands for Asylverfahren, i.e., asylum procedure) and the organisational section (No. 6). 
Membership moreover literally means having a 'place' to enact the dispositif. It is strongly related to the key inhabited space of administrations: the office. It is at the same time a highly standardised space and a personalised space. ${ }^{8}$ I noted a key division between officials having their own office and those sharing an office with others. Having an individual office was highly valued by caseworkers in the headquarters. While seniors and long-term employees usually had their own office, most newly hired caseworkers, some part-time employees, and the secretaries had to share an office with usually two to three others. The most important advantage of caseworkers with a personal office is that they can conduct hearings in their rooms, while those in shared offices need to go to other rooms for the hearings, sometimes in other buildings. In contrast to the limited and thus contested working space in the headquarters, the office situation in the Reception and Processing Centre where I did field research was not an issue (at that time). All caseworkers had their own personal offices there.

Membership devices are crucial for agentic formations of caseworkers: they consist of badges, keys, smartcards, acronyms, but also allotted office space and time to work from. While such devices appear trivial, their mediating role becomes apparent when they are lost, ambiguous, or contested. They, in a crucial way, enable caseworkers to become bodily and virtually part of various spaces, times, and circulations - outside of which case-making is hardly possible.

\subsubsection{Super-Vision}

I use the term "super-vision" to describe how some people are able to see more than others in the office - and have a different associative capacity in terms of the dispositif. It is usually associated with people with higher positions who have influence over decisive matters. A caseworker, with whom I had attended the basic training and interviewed a year later, had just been appointed as vice-head of his unit. He told me about why he sought this promotion:

8 Ordered office space (on different levels - buildings, offices, desks, shelves, archive, forms) and ordered office time (regular working hours, sequenced tasks, appointed meetings and completions) both allow for and mirror systematic and orderly administrative activities (see Valverde 2014). 
To make a career in this sense [was not central], I mean it is nice to see you get on somehow and are able to develop yourself. But for me it was more that I had the feeling, if you are a bit higher [in the hierarchy] you see a bit more. I mean you see this yourself if you do research about this: that's intricate, this whole business, right? That's a huge cake [Riesenkuchen], and to be in the know [den Durchblick bekommen] is not that easy. And I had the feeling, if you are in a leadership position, you see a bit more. And I really think I have already figured out two or three things that I did not understand before. (Interview with vice-head of section, autumn 2013)

When I asked him what he had figured out now, he said "procedural stuff" and added:

before [my promotion] it virtually ended for me with having conducted the hearing or the decision* written, right? Now it has become interesting ... what happens to the case file after a decision*, what happens to the people themselves after such a decision* and that sort of thing. (Interview with vice-head of section, autumn 2013)

His new position only comprised part of his employment, and he emphasised that he was still conducting hearings and writing decisions alongside his new tasks. What these quotes highlight is the peculiar vision (but also "myopia", see Whyte 2011) that comes with hierarchical positions in the office. While in this particular case the vision still remains closely associated with assembling cases, there is arguably a certain disjuncture in vision between the heads of sections (and to some extent of divisions) and the upper management not directly in touch with casework. The upper management 'sees' casework to a considerable extent through statistics, as officials engaged in casework on various occasions suggested (and I discuss in sections 8.2.12). Aside from work statistics, it attempts to conduct caseworkers' conduct through quality charters, internal guidelines, recurrent training sessions, information emails and events, and the promotion of a good "office culture" [Amtskultur] (which, for instance, headlined an issue of the internal magazine of the SEM, the PIAZZA in 2015).

Inside the 'productive sections', super-vision consists of much more than seeing casework through numbers. The association between caseworkers and their heads of sections (which also refers here to vice-heads) is key for 
case-making. In the sections in which I did my fieldwork, the role of heads of sections - as the word "supervision" indicates - encompassed both oversight and assistance. Heads of sections monitored case-making quantitatively as well as qualitatively, and they could be consulted in any question concerning casework. They also gave novice caseworkers written or oral feedback on their decision" drafts.

Probably the most important technique for directing the quality of casework, namely the content and form of decisions", is the so-called "four-eye principle" [Vieraugenprinzip]. ${ }^{9}$ Formally, the four-eye principle implicates clearly defined responsibilities: caseworkers determine the facts of the case" and write a decision* proposal [Enscheidantrag]; (vice-)heads review and authorise or reject the proposal. The latter confirm with their signature that the case was processed respecting the binding quality criteria and that the decision" conforms to law, internal instructions, and the asylum practice*. In practice, it means that the head or vice-head of the section has to see important records before they are issued and confirm this inspection with their signature in the right place in the document. Such an acknowledged inspection is compulsory for asylum decisions" and some laborious and expensive mandated investigations (such as LINGUA tests and embassy inquiries). While the depth of inspection by the superiors remains off-record, their signature on the document is testimony to the document having 'passed their desk'. Additional to the mere 'control' of important records, it serves to distribute responsibility for what could be implicating serious consequences (see section 8.1.1).

The heads of section I spoke with all looked with variable scrutiny at the rulings (and other important documents) depending on the experience and (perceived) reliability of the caseworker who had written them. They had a sort of sampling system of how they picked out caseworkers' decisions to scrutinise them. The reception and processing centres used a similar system in regard to protocols of first hearings. A head of section in the headquarters employed what he called "red flags", a form of heuristic for the selection

9 Other crucial figures are relief organisation representatives in hearings who have an important function in the 'monitoring' of main asylum hearings. With the new procedure introduced in 2019, legal representatives will attend both first and main hearings and comment on decision* drafts and thus become key not only for the monitoring of hearings but also concerning asylum decisions*. 
of cases, for which he always examined the decisions more closely: those of unaccompanied minor asylum seekers and single women, and of caseworkers who (in his eyes) were struggling. But, he added, how closely he would look at the decisions largely depended on his capacities and how "in form he was that day" [seine Tagesform] (Fieldnotes, headquarters, winter 2013/14).

In their inspection, head of sections rarely overturned the type of a decision* itself, but rather the argumentation to corroborate it. ${ }^{10}$ As a caseworker told me:

It's more about the argumentation, I think. That they [the heads of section] either say: the argumentation does not withstand or argue differently or more clearly, structure it differently. That's what I was blamed for two or three times: structure it differently, this won't work out like that; well, the argumentation works but build it up differently. Yes, and the argumentation [with Articles] three and seven he also told me once, I guess. Or he enquired further: why do you argue with [Article] seven not with [Article] three, that would actually be a clear three. By trend, in our section, it is not virtually a command, but it is rather - because you are the one who knows the case best-it is rather a question, why do you make it that way? And if you then can argue reasonably, then it's actually ok. Furthermore, very banal: mistakes in writing are corrected. (Interview with caseworker, autumn 2013)

Overall, the inspection of head of sections can consist of a thorough (proof-) reading and commenting of the argumentation in the decision. But the inspection may also be limited to flicking through the document and appending a signature. Most superiors acknowledged that this meant they occasionally missed something. Yet they emphasised that, like the caseworkers, they had to economise their time resources.

Importantly, this account of super-vision in the asylum office implicates that there is no "god's eye view" (Haraway 1988), neither in an administration. All vision is situated and necessarily partial - however, super-vision also implies that views may be crucially connected. In effect, super-vision also means that "life and death decisions" (Douglas 1986, 111) do not place a

10 This can be read as a sign of trust of superiors, but could also be an effect of caseworkers' anticipation of what their superiors consider the 'right' approach (for certain types of cases at that time). 
heavy responsibility on the shoulders of a single human: they are always at least shared between two (well-equipped) officials - the caseworker and the superior. ${ }^{11}$

\subsubsection{Re-Collecting Collectives: Meetings and Minutes}

Meetings are key events for the asylum office and its subdivisions as moments in which they constitute themselves as collectives. I could participate in meetings on various occasions and in various assemblies: regular team meetings of sections, but also a range of other meetings such as information and training events and meetings to develop the practice* (for an example, see section 7.2.3). A key part of the weekly team meetings of the sections consisted of the head or vice-head of the section summarising the key issues which had been raised in the upper organisational bodies' meetings; besides, the minutes of these meetings were circulated amongst all employees of the asylum office (at least of the headquarters); and in some sections, important sections were directly copy-pasted into the minutes of their meetings. ${ }^{12}$ Communications from the upper bodies could consist of both directives affecting casework and reporting on upcoming issues and on-going projects to inform employees about what was decided and planned 'above' and in special working groups. They could also contain upper bodies' acknowledgements of problems raised by the caseworkers themselves and actions envisaged or already taken to resolve them. Here's a glimpse of the scenery of a section meeting I attended:

All of the caseworkers (except three) of the section are gathered in the unadorned meeting room around tables arranged in a large oval. Also present are the secretary of the section, who writes the minutes, and the vicehead of the section. Thomas, the head of section, sitting on the short end of the oval, opens the meeting and welcomes everyone. He will not comment on

11 While the four-eye principle prevents caseworkers from resolving cases single-handedly, it seems not able to alleviate the tendency that no one feels responsible for a case anymore arising more generally from the division of labour in case-making (Eule 2013; see also section 8.1.4).

12 I was thus in various ways informed about the activities of other organisational bodies (particularly the executive board [CL-Ceschäftsleitung], the steering committee [PILAR] and the heads of division [ABR-Abteilungsrapport]). 
the minutes of the executive board's latest meeting, he says. At the management retreat [Kaderklausur], he briefly states, it was decided to shift bonuses from small bonuses for everybody to large ones for only a few employees. They moreover discussed leadership principles and Kristina gave an awareness-raising input on human trafficking. Bernd, a caseworker, asks Thomas how they should take the statements of the director [of the migration office] about the work of the asylum directorate regarding its production the had expressed his concern about the low production despite considerable new staff having been hired]. Thomas responds that he [the director] is also under strain from above [Federal Council and Parliament] - he therefore had to see where one could become more productive and save time. (Fieldnotes, meeting, headquarters, winter 2013/2014)

In meetings, the sections and divisions take form: they are assembled, in every sense of the word. The bodily encounters in meetings add an experiential grasp of belonging for 'communities' which are otherwise brought to existence on organisational charts, in mailing lists and identifiers and spatial allotment in office buildings. Administrative meetings are highly scripted encounters - featuring a particular spatiotemporal arrangement, a set of agenda items to be completed, assigned roles of speech, and a particular form of textual outcome. Sometimes the scripted nature of meetings expresses itself, as in some instances, the announcement of agenda items for the meetings already have the form of provisional minutes. In these cases, the scripting and recording of meetings coincide to a considerable extent. However, I would not infer that such meetings are necessarily more strongly scripted in practice than others; rather, they more openly disclose the script of the meeting. For many parts of meetings, speech is clearly assigned. The person leading the meeting opens and closes it and guides through the agenda items. Other persons will receive the permission to speak on certain items, for which they prepared. On certain items, however, participants are explicitly asked about their opinions and assessments. Moreover, they can raise questions concerning more scripted items and concerns about issues and put things troubling them on the agenda as well. This happened in the meeting I started describing above:

On the section level, he mentions that he discussed a suggestion by Wenger [the head of division] with Carla this morning, which they should turn into a 
report by, hopefully, next Monday. He does not want to say anything specific about this. But when a caseworker asks him about the suggestion, he still explicates a bit more: it is envisaged to conduct accumulative hearings ${ }^{13}$ for Eritrea, similarly to those successfully employed for Nigeria. A murmur goes around the 'crowd'. A caseworker objects that this could have consequences for the numbers of applications filed from Eritrea, because currently their proceedings take so much time in Switzerland that they prefer to move on and file an application in Sweden. He asks whether the executive board has considered this. Thomas negates this and adds with an expression of mild sarcasm that as very often the management hasn't been concerned with this, the consequences remain uncertain.

Kristina asks how this should actually work in practice. They always speak of improving efficiency and now: accumulative hearings. Yet for two months now they have to pick up applicants arriving for hearings in the Q6 [the main building] before going to the hearing room, which takes them about a quarter hour. If they only make one break per hearing (in the main building), this sums up to a whole hour of travel. Bernd joins in to state that this is really cumbersome. Thomas asks whether it would make a difference if applicants could register directly in the Q17 [the annexe building hosting the bulk of asylum caseworkers]. (Fieldnotes, meeting, headquarters, winter 2013/2014)

Remarkably, a silent agreement seemed to prevail in administrative meetings I attended about what parts of the meeting would be on- and off the record. Minutes are crucial, not only in public administration, for every formal meeting. But I suggest it is exactly the mundaneness as a seemingly natural artefact of re-presenting meetings which makes it worth pondering on them a little bit. The minutes are the only written recollection of meetings and bear different roles. On a first level, they serve to record organisational acts and participation. They perform organisational associations by officially yet internally transmitting news, decisions, and specifications 'from above'.

13 In contrast to the common organisation of hearings - an applicant is allotted a half-day slot with the according 'team' for conducting the hearing - in accumulative hearings, a number of applicants are invited for their hearings on the same day and then queue for their hearing to be conducted by one of the 'hearing teams'. This form of organising hearings is considered more resource-efficient, but only works for countries of origin with many applicants and rather short hearings on average (Fieldnotes). 
They are both retrospective and prospective: they capture what happened and they announce what is going to happen in the office and its (relevant) subdivisions. They serve as a classifying tool to distinguish between the inside and outside of the meeting, between the said and the unsaid in relation to the record. As a textual artefact of office-making, they have an afterlife and circulate inside the office via email distribution lists and in print, and they are archived on a document server for future reference. They are important elements in citational practices within organisations (Butler 2011), performing belonging and hierarchy and enrolling participants in common action (Callon 1986). They are both intra-referential by citing earlier protocols of this collective, and extra-referential by citing protocols of others (e.g. the management board) and other sources (such as leading decisions from the Federal Administrative Court).

An analysis of my fieldnotes on the different meetings of productive sections I participated in revealed the following five recurring elements of such meetings: awareness-raising and preparation, direction, b/ordering, lobbying, and practicalities.$^{14}$ Meetings serve for awareness-raising and preparation. Caseworkers are introduced to the larger developments related to their work such as upcoming legal revisions, the consequences of novel legal provisions and of changes in country or thematic practice" for case-making. They are informed about mandatory and optional upcoming events on various levels of the office and beyond. Moreover, they are prepared for organisational reforms, including human resources issues which may affect their posts or requirements (e.g. agreements on objectives).

In meetings, caseworkers receive direction for their work as priorities are made explicit. They are reminded of principles, standards are introduced, and more. The quintessence - in the eyes of the superiors - of new regulations is enunciated. Both written guidelines and verbal heuristics are offered to caseworkers for their everyday categorisation work. Regarding the question of how to increase the output, a superior suggested in a meeting to not be overly formalistic and perfectionist:

The superior asked for a pragmatic stance: if [considering an asylum appeal] the Federal Administrative Court asks you for a clarification on various

14 Overall, I participated in more than a dozen meetings of four different sections including the reception centres. 
points, which imply a temporary admission, you should not do the 'exercise' but make a temporary admission right away. (Fieldnotes, headquarters, winter 2013/2014)

Meetings are key sites for b/ordering. They provide authoritative 'readings' of what is happening. A crucial element of meetings is the qualification of performance: the output accomplished in, or production expected of, the unit. Such 'numbers talk' often offers a relational qualification - how is the performance relative to other sections, targets, or the past (including justifications for differences amongst sections or targets not reached)? Furthermore, meetings are used for the b/ordering of competence: for stating what is within and beyond the scope of sections, and relatedly the delegation of work upwards and outwards. Crucially, meetings are sites of identity formation through the mobilisation of manifold discursive Othering practices (see Said 2009). A difference between 'us' and 'them' is erected through the distancing of suggestions or practices of others, or blaming them for problems (see section 8.1.3). Caseworkers are sworn in to the views and approaches of this particular section, and also its established ways of coping with difficulties. They moreover learn about their superiors' taste and matters of preference. And meetings can offer some room for superiors to display their own discretion in the implementation of orders from above by instating small acts of resistance or calling for disobedience regarding measures from above they consider unnecessary or detrimental. They offer some room for joking about irrationalities of the system they have to deal with:

The superior said with an ironic undertone that it's nice that all ... employees of the migration office receive the minutes of meetings of Cattiker [the director] over email; but the important things (...) they only hear via the media. (Fieldnotes, headquarters, spring 2014)

A further key element of meetings that I identified is lobbying: meetings offer a common space for raising problems, for example concerning working conditions; they are also an open space for discussing or dismissing solutions. This becomes obvious in the continuation of the meeting above:

Kristina raises the list of deficiencies she had prepared, sacrificing a whole morning, but this would probably be neglected. The security in Q17 is alarm- 
ing, she says; if the people are in the waiting room, nobody monitors them anymore. They could easily get into the main wing of the building. Furthermore, the applicants and the interpreters and relief organisation representatives are all in the same room. We don't have to do hearings anymore if they can conspire beforehand. Yet, there is a second waiting room next to it that is unused. And to add to the chaos in the corridors of the Q17, one wants to conduct accumulative hearings... Thomas apologetically chips in that the issue with the side entrance [reserved for officials] is still pending in a committee. These just are not decent working conditions, Kristina indignantly stated. (Fieldnotes, headquarters, spring 2014)

Sometimes, meetings seem to have the function of a pressure valve for caseworkers, where they can voice anger and protest and be heard.

A last aspect of meetings are practicalities: superiors or other caseworkers suggest workarounds to deal with everyday problems or introduce heuristics or tools for simplifying things. In sum, meetings are key sites for assembling and perpetuating communities of meaning (Yanow 2003a) by offering frames for interpretation, authoritative readings and an arena for participation and negotiation. They moreover provide means for formatting communities of practice (Wenger 2003) by stabilising classification systems through re-configuring heuristics, offering new exemplars, and defining what matters. ${ }^{15}$

In this subchapter, I have suggested considering the complex agentic formations of caseworkers: as 'lone', yet also crucially as 'collective warriors'. Their individuated agency takes shape through their equipment with devices for accessing and enacting (physical and virtual) space-times of case-making. Their collective agency arises, for instance, in associations of super-vision and meetings which enrol them in assemblies of case-making. For such assemblies to become agentic, however, a transcendental figure - here, the nation-state that (allegedly) delegates them the duty to resolve claims of 'applicant others' and thus authorises case-making - must be invoked.

15 I do not discuss special committee meetings here. They occur quite rarely, but have often far-reaching consequences for case-making, as they shift the scope of notions and technologies; for instance, doctrine meetings [Doktrinrapporte] (re-)forming a specific asylum practice* (see section 7.2.3) or country-specific situation assessment meetings [Lagebeurteilungssitzungen]. 


\subsection{Technologies for Assembling Cases}

Documents promote control within organizations and beyond not only through their links to the entities they document but through the coordination of perspectives and activities. (Hull, 2012, 257)

In this second subchapter, I focus on the material-discursive devices that enable casework. I follow Hull (2012a, 259), who suggested to attend to the "generative capacity" of documents, as they "are essential elements of the constitution of a vast variety of entities", in my case asylum and its subjects. Considering the material politics of asylum, I treat bureaucratic documents and other socio-technical devices as mediators, "things that 'transform, translate, distort, and modify the meaning or the elements they are supposed to carry" in order "to restore analytically their visibility" (Latour 2005, 39). Latour and Woolgar (1979) introduced a now-famous type of mediator in their laboratory study: the "inscription device" that refers to a thing (in their case an item of the laboratory machinery) able to "transform pieces of matter into written text" (Latour and Woolgar 1979, 51). Such devices "can transform a material substance into a figure or diagram which is directly usable by one of the members of the office space" (ibid.). They thus provide simply 'readable' traces - inscriptions - of a complex laboratory experiment, which are "regarded as having a direct relationship to 'the original substance" (ibid.), yet omit the intermediary steps of their production (ibid., 63). Such inscription devices not only exist in laboratories (Law 2004b, 7), but also, as I suggest, in administrations. However, as Law $(2004 \mathrm{~b}, 9)$ pointed out, inscription devices are not the only devices for enacting realities; thus, we "should be trying to discover and characterise what we might think of as alternative enactment devices or modes of enacting". These material-discursive devices have to be conceived as elements of governmental technologies which comprise "the forms of knowledge, skill, diagrams, charts, calculations and energy which make [their] use possible" (Barry 2001, 9). I thus suggest considering a range of technologies involved in the enactment of the asylum dispositif and their respective devices. I distinguish them according to the work they do in recording (5.2.1), inscription (5.2.2), coordination (5.2.3), and writing devices (5.2.4). They do not 'act' on their own, but mediate practices of case-making and thus crucially (re)shape the ways in which lives are recorded through the asylum dispositif. 


\subsubsection{Recording Devices}

Governmental technologies of recording assemble and 'hold' things pertaining to a case together and allow them to speak forcefully as records [Akten]. Crucial technologies of recording are filing and pagination. Recording devices help transforming documents of various sorts and origin into records. Key devices for the unequivocal attribution of records to cases are, for instance, case files, numbering, and file registers. Signatures, stamps and seals render records to be the 'bearer of rules' and provide "a spectral presence" of the state (Das 2004, 250-51), i.e., one capable of re-cording lives of those they enrol.

Figure 5: Desk with case files in the headquarters

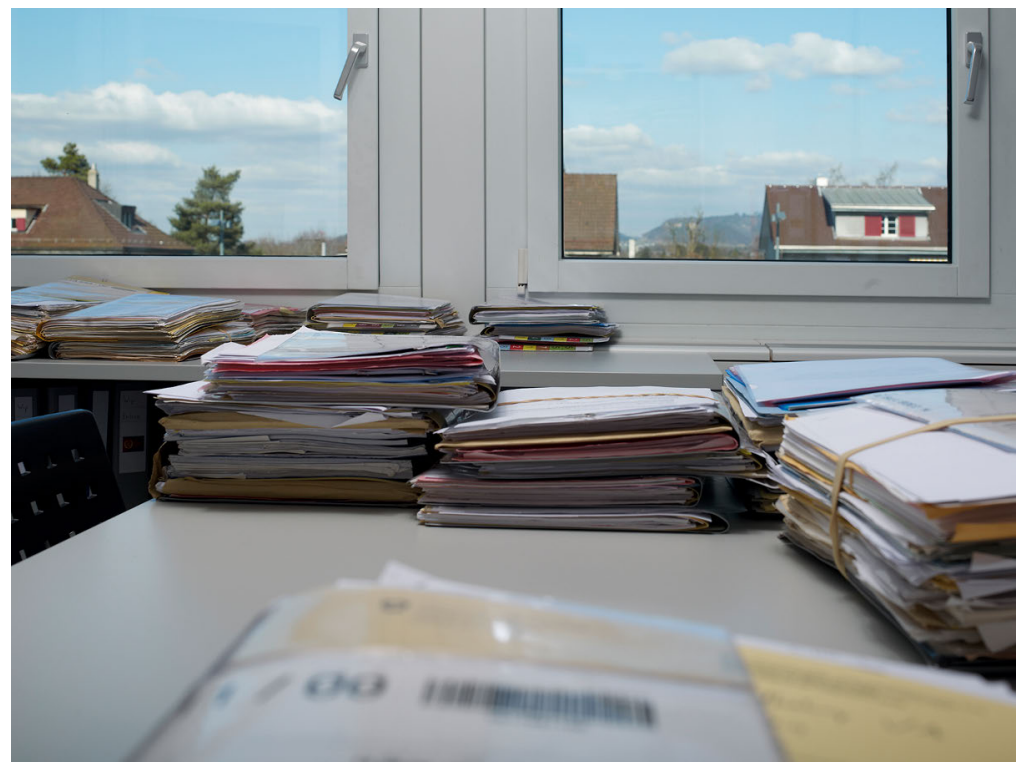

(Source and Copyright: Dominic Büttner)

When I first entered an office in the asylum administration, I was drawn to the omnipresent case files of varying thickness populating desks and shelves (see Figure 5). From afar, only the colourful, six-digit $\mathrm{N}$-numbers protrud- 
ing their covers were visible. ${ }^{16}$ This number not only labels the envelope of the case file but also marks all records pertaining to it. It is automatically inserted in the standard letters written for the case and added by stamp or in handwriting to, for instance, materials submitted by the applicant to enter the file. Case files are often complex composites, as they hold all the federal immigration-related application files of members of a core family (spouses and minor children are bundled in the same case file) and also non-asylum files with records on immigration-related applications (for instance regarding visas, return assistance or family reunification). This means, on the one hand, that case files also circulate and become assembled partly outside the asylum divisions of the office. On the other hand, even though such additional files may not be directly relevant for the asylum application, they still mediate how caseworkers encountering the case 'see' it. Case files may grow to a size of more than one volume with second applications, applications for re-examination, or applications by different members of a core family.

In the reception centre, I received for the first time a case file to inspect and had a closer look at its composition:

The whole morning, I plough through the seemingly interminable case file, which consists of two application files (yellow), between which an enforcement assistance/ZEMIS ${ }^{17}$ file (green) and some loose emails and partial prints of a ruling of the Federal Administrative Court (FAC) are scattered. On the backside of the latter, handwritten headwords of argumentation fragments are listed, on whose basis the decision* will likely be assembled. They differentiate between an argumentation with Article 3 and Article 7, respectively. Means of evidence are collected in brown envelopes in the application files, which are provided with a register of their content. On the cover of the application files, the chronology of records inside is listed. Moreover, it is indicated by a code letter, which records an applicant can inspect on request. I go through the records from the back to the front, which means

16 Every numeral has a specific colour for simple recognition. The initial letter "N" has historical roots and stands for Cerman "Niedergelassene" - "established person" - although this does not make much sense for asylum applicants. I was told that before the $\mathrm{N}$ was introduced for all foreign national dossiers in 1936, case files had a "P-number" (for Cerman "Person").

17 ZEMIS is the abbreviation of the central migration information system (Zentrales Migrationsinformationssystem) (see also section 5.2.3). 
from the beginning to the end: records are filed in reverse chronology, with new records added on top of older ones. Thomas had asked me just not to make a mess with the content - I try my best. Roughly, the case file consists of a first application from 2011, which the Federal Office for Migration (FOM) concluded in the same year with a DAWES $32.2 \mathrm{a}$ and which also the FAC confirmed. About half a year after the first decision* came into legal force, the applicant filed a second application. The FOM wrote a DAWES 32.2e decision [a formal rejection of application due to previously unsuccessfully traversed proceeding without leaving the country and without new reasons]. However, in the second application the applicant had furnished two newspaper articles as fresh evidence, which the FOM had not considered substantively. The FAC therefore scrapped the decision* and determined that fresh evidence needed to be assessed substantively. In February, another hearing had been conducted by the FOM, and the decision* is now pending. The file came here to an end and so did my inspection. (Fieldnotes, reception centre, winter 2012/13)

Besides offering a glimpse into the content of this single case file, the inspection reveals a few more general aspects of case files' composition: case files are inside partitioned into files (at least one), which are again filled with records. ${ }^{18} \mathrm{~A}$ common and fairly minimal set of records in an asylum application consists of the personal form, the triage form (a form that summarises and categorises the case and accordingly stipulates further steps of processing), protocols of two hearings, and an asylum decision*.

These records are listed on the cover of such files in the file register [Aktenverzeichnis] (see Figure 6), another key recording device. Already from this register, one can therefore recognise the records officially assembled in the file so far. The file register moreover indicates the stage of an application. In the file register further associations are established: a keyword characterising the record (for instance "protocol of first interview") and the acronym of the person registering records and the date are specified. Finally, a letter (A-E) prescribing whether or not a copy of the record is to be released with the decision* or in case of an application for the inspection of records [Akten-

18 Files for different proceedings are distinguished by their colour-yellow files containing asylum applications. 
einsichtsgesuch] is prefixed to the record number. ${ }^{19}$ Through this classification, some records are rendered 'out of reach' for claimants and their legal representatives outside the administration. File registers are thus recording devices that allow the things that belong to a case record to be assigned to them in a well-ordered, sequential, and referable form. They are the central devices in the governmental technology of pagination.

Figure 6: File directory of asylum case

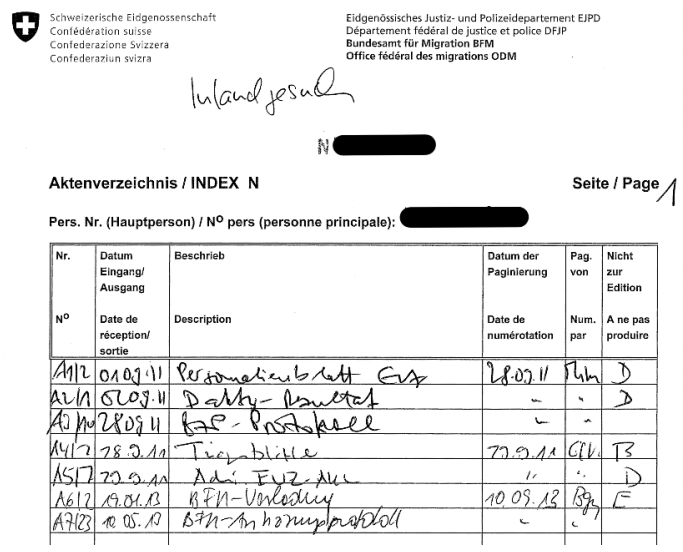

(Source: Fieldwork materials, winter 2013/14)

\section{Provisional records}

Usually, not all of the documents pertaining to cases in the making are registered yet, but the newest ones are loosely assembled inside, waiting to be rendered official records. Sometimes documents' transient state is related to their unsettled status when they are not ready to be officially registered: for instance, when the control and signature of a decision* is yet missing. In this case, they end up on a pile, with the document to be checked attached with a paper-clip outside to the cover of the case file. For reasons of efficiency, documents are often only registered before the case reaches a certain stage, for instance just before they are passed on to another caseworker or section (see subchapter 6.3). Transforming provisional records into official case files

19 The legal provision regulating inspections of records in administrative procedures is found in Articles 26-28 of the Administrative Procedure Act (APA). 
records occurs through a technology referred to as pagination [Paginieren]: it inscribes and thus stabilises the association between documents and case files; the former become records of the latter. New caseworkers are introduced to pagination work as a part of their training in the sections. A short excerpt from my fieldnotes shows how I learnt about the key distinctions to be made regarding the classification of records:

We go with Katharina, the mentor of the new caseworker I could accompany, through the classification of records. A-records are those of "public interest", the release of which could give someone valuable clues about confidential issues and cause harm to Switzerland: one would classify an embassy enquiry or LINCUA expertise as A. B-records are internal documents, for instance file notes or triage forms; but Katharina said to us that we could also put a $D$ in these cases if they do not provide anything new. She advocates for common sense in this respect. C-classified are records produced by other authorities, often the canton or the municipality (for instance the civil registry office), which the Federal Office for Migration receives in copy. D-records are "inessential records" (from a procedural point of view), a typical example being asylum seekers' transfer permit from the reception and processing centres to the canton. E-records, finally, are records known to the applicants: either documents or evidence submitted to the office by applicants or their legal representatives or documents sent to them or their legal representatives by the office (for instance the summons to the hearing). Upon request for the inspection of files (after the investigation has been concluded), the office provides the applicants or their legal representative with a compilation of all non-classified records in the file. Upon explicit request, records classified D or E would also be provided. (Fieldnotes, headquarters, spring 2014)

In the training, we were further given an "internal directive on the creation, management and editing of procedural records"20 containing a long (and somewhat outdated) list of records that could potentially enter a file with the appropriate classification for each of them. During pagination, some documents are filtered out that are not envisaged for the record (such as personal notes and interview guides). While the classification of records can be challenged in the appeal court, the removal of records that could tell something

20 In German: "Interne Weisung über die Anlegung, Führung und Edition von Verfahrensakten". 
about the considerations and events through which the case has emerged is definitive. Records' existence is at stake in such prosaic pagination practice, as Vismann $(2011 \mathrm{~b}, 102)$ rightly suggested, since "what shall be included in the records ... is contested". Records' ability to speak in subsequent citational practices (Butler 2011) varies considerably. Some of the records are only able to internally whisper (through their classification as "internal" or "confidential"), but may nevertheless remain forceful in speaking for or against protection or expulsion of a claimant. Other records (such as the hearing protocols) are made to speak more publicly and are extensively cited in the asylum decision". The classification of records thus determines where and how they are able to speak.

\section{Excursus: Delivery fiction}

Asylum applicants are usually ${ }^{21}$ informed about the outcome of their claim with a registered letter sent to their housing address containing the asylum decision". The applicant has to has to confirm the reception of the registered letter with a signature on the return receipt [Rückschein]. The date of reception on the return receipt is crucial because it determines the beginning of the statutory period for an appeal against the ruling. If the letter cannot be delivered but is fetched at the post office, the date of collection marks the beginning of the statutory period. A copy of the doubly signed and registered letter delivering a ruling enters the case file as a record. If the letter cannot be delivered and is not fetched at the post office, the unopened envelope is returned to the asylum office. As a record in the case file, it represents a failed notification attempt. The letter is directly returned to the asylum office, for instance, if the address of the applicant is for some reason incorrect. However, if the address is correct and the letter is not collected at the post office, the letter counts "at the seventh calendar day following the unsuccessful delivery attempt as delivered" (Fieldnotes, basic training for new caseworkers, autumn 2012) - a legal construct called 'delivery fiction' that also applies if the reception of the letter is refused. The fictitious event of order delivery is recorded in the case file to induce the statutory period for an appeal. In sum, the "delivery fiction" of asylum decisions" points to an interesting difference:

21 Decisions are, rarely, disclosed to the applicants in oral form as well. (This applies potentially to all types of decisions in the reception centre but only clearly positive decisions in the headquarters.) 
what counts as a delivery is amounts to more than what is effectively delivered. This legal invention resolves the problem of decisions* becoming legally effective if the applicant cannot be notified about it. It responds to the recurring problem of applicants absconding before the end of their procedure. While the significance of stamps and signatures has been widely acknowledged (see Das 2004; Hull 2012a; Mathur 2016), the delivery fiction can be considered as an example of a technique to render a provisional record 'official' without the otherwise essential signature.

\subsubsection{Inscription Devices}

What I call "inscription devices" are a rather heterogeneous set of material-discursive devices that make 'outside realities' amenable to the case. They converge in their purpose of producing what I introduced above as the "facts of the case" necessary for resolving it. But they have - as for the inscription devices described by Latour and Woolgar (1979) - the characteristic of removing the complicated procedure for their production from view. They are readable and citable in other records - most importantly asylum decisions* - but also beyond the case. Two core technologies for 'importing' realities into cases are discussed here: hearings and further clarifications. Hearings produce key records that associate the case with the applicant's life - or, more precisely, they import a particular version of lives into cases through the inscription devices called protocols. ${ }^{22}$ Further clarifications produce certain supplementary renderings of 'realities out there' to be imported into cases. Such clarifications comprise linguistic tests, document checks, consultations, embassy enquiries, medical reports, and supplementary hearings. ${ }^{23}$ Below, I will briefly introduce linguistic reports as the probably most common of these devices (an embassy enquiry and a consultation appear in subchapter 6.4). Instances of such technologies of inscription and their devices that I introduce here are (a) hearings as a form of testimonial interviews to produce protocols extensively cited in decisions* considering

22 Hearings are a typical example of a technology involving more than one kind of devices. Various coordination devices crucially script them: forms, interview guides, and so-called APPA (see section 5.2.3).

23 Further enquiries also need to be registered in the code at the end of the decision because they are statistically relevant. They thus count in a particular way and are grasped by 'coordination devices' (see section 5.2.3). 
the "facts of the case" and "credibility"; (b) country of origin information (COI) that produce 'facts' about certain places at a certain times that can be compared with accounts captured in protocols; and (c) linguistic tests that produce LINGUA reports as 'expert accounts' of applicants' origins.

\section{Hearings and protocols}

Hearings are a quintessential technique for case-making: they allow the association of cases with applicants' statements, which they both elicit and inscribe. For case-making, two hearings are standard: a first hearing conducted in the reception centre and a main hearing typically conducted in the headquarters. The first hearing is a rather structured hearing internally referred to as BzP (Befragung zur Person), which literally means "enquiry about the person". It consists of filling out a form with questions focusing on the person: the applicant's identification by reference to nationality, residence, age, family, identity documents and the travel route from the country of origin to Switzerland. Applicants have to briefly state their reasons for asylum as well. ${ }^{24}$ The second, main hearing [Anhörung] focuses explicitly on the reasons for asylum: it is less structured - usually only conducted with an interview guide developed by the caseworker. It is supposed to leave enough room both for a "free account" [freie Erzählung] of the central persecution story that led to the applicant's flight. Another core part consists of the caseworkers' enquiry into any aspects of this story that appear implausible or unclear with respect to their relevance for asylum..$^{25}$ The two forms of hearings are different regarding the participants: in first hearings, usually only an applicant, a caseworker and an interpreter take part; in the main hearings, a relief organisation representative and minutes taker are also present. Both hearings are protocoled and materialise in printed form at the end. Before becoming records, they are retranslated for the applicants who have to sign each page to confirm that the protocol properly represents what they said. Finally, the caseworker and the interpreter sign on the last page. The relief organisation representative's form with potential 'objections' to the conduct

24 Caseworkers can amend questions in the form to any of the categories if they consider doing so necessary for the case. Such a first interview usually takes between one and three hours.

25 Such main hearings are - depending on the complexity of the case anticipated from the first hearing-usually either scheduled for half a day or a whole day. 
of the hearing is attached at the end (see subchapter 6.2). In institutional terms, such records contain what are called "on-file facts" [aktenkundige Tatsachen], i.e., "fragments of written texts [that] can be used for reasoning decisions" (Affolter 2017, 48). Such fragments contain thus the bits and pieces required for case closures (see subchapter 6.5).

In the frequent absence of material proof or evidence for persecution and origin, hearings are the key technology for producing "the facts of the case" that become citable through the inscription device of the protocol (Presentation handout, basic training, 2012). In the vast majority of cases, protocols provide the crucial associations to the 'outside reality' required to resolve them. But they have another purpose: they serve to gather information which can be taken as an indication for or against the credibility of applicants. According to the basic training for new caseworkers, an essential function of hearings is thus the "production of usable statements" (Presentation handout, basic training, 2012). A range of techniques and devices are involved in this production. New caseworkers were advised to prepare main hearings thoroughly by studying previous records (certainly the protocol of the first hearing), considering the threat profile (the persecuted person, persecutor, persecution motivation, persecution event(s)), consulting country-specific background information, and developing a written interview guide. During the hearing, they need to "ensure that the applicant makes usable statements to the topics relevant for the decision" (Presentation handout, basic training, 2012). For this purpose, caseworkers were introduced to particular techniques for asking questions (e.g. moving from open-ended questions to targeted questions and, if necessary, closed questions). Hearings can be analysed in the way they are conducted as a form of testimonial interview (Scheffer 2007b). The double role of hearings as a technology for the "neutral gathering of facts" and the "production of usable statements" can be read, according to Scheffer (2007b), as the "duplicity of testimonial interviews" (see section 6.2.3). Hearings can moreover be analysed in terms of the process of "entextualisation" (Jacquemet 2011), of turning speech into text detached from the social context of its constitution. For the latter process, protocols operate as crucial inscription devices.

A crucial facet of hearings is that the 'right questions' are asked and find their way into the protocol. In the basic training, the senior official teaching the module on "credibility assessment and hearing technique" shared some of her heuristics with new caseworkers: 
It's wrong to protocol feelings. But it's good address feelings by for instance stating: "I realise that you are very amused". It's important to address 'strange' emotions. They can tip, from laughing to crying, for instance. Sometimes applicants try to overplay how affected they are by what happened: this means you should not assume someone is lying because he laughs! But have the courage to enquire - it's not bad to look stupid. In the end, it only counts what is in the protocol. (Fieldnotes, basic training, 2012)

This is just to give a glimpse of a whole range of heuristics and techniques that caseworkers I encountered have suggested regarding the conduct of hearings. I came to see hearings as a complex technology considering the knowledge and skills it takes to conduct a hearing, but also because it involves the interplay of human participants (interpreters, relief organisation representatives and minute-takers) and non-human participants (namely protocols, interview guides, computers and printers). As Latour (2005) highlighted, such participants may all have an (unforeseeable) effect on the course of such events as forceful mediators. A group of experienced caseworkers with whom I discussed questions of discretion told me in a coffee break that they think the work of conducting hearings "is highly underestimated" in the office (Fieldnotes, headquarters, spring 2014). They did not imply that their work is not appreciated, but that both the management and most caseworkers do not sufficiently admit the complexity and full scope of asylum hearings in their view.

\section{Country of origin information (COI)}

Imagine you have a Belarusian who says that he's part of an anarchist group and he is thus threatened with imprisonment upon return to Belarus - which counts as the last dictatorship in Europe. Then you will need country of origin knowledge: $\mathrm{COI}$. (Fieldnotes, basic training for new caseworkers, autumn 2012)

The production of so-called country of origin information (COI) is a crucial technology for generating more or less generic and authoritative 'facts' against which the statements of applicants can be compared. Caseworkers may draw upon COI to "objectify" applicants' fear of persecution (Barsky 1994; Gibb and Good 2013, 292; Popovic 2005) and unmask 'bogus' accounts. 
It is consultants of a specialised country analysis unit of the asylum division who produce such knowledge about applicants' countries of origin.

Country analysis, as the practice of producing any type of COI, draws upon a range of technologies and inscription devices for producing authoritative knowledge about the world 'out there'. A country analyst introduced her work in a module of the basic training. She introduced what country analysts produced: "written products" from things such as consultations for individual case clarifications, but also extensive reports about certain topics, for instance about the medical situation in a country, and more comprehensive country reports. Analysts also compile information for the internal COI database (reports, maps, statistics from other sources). She suggested that for individual case clarifications, we should first consider collecting the information needed ourselves, because analysts were often working to capacity. She gave us some advice for ascertaining the quality of sources used:

The quality of the sources is essential. But how do we evaluate the information we find? Probably many of you still know about this from university, source criticism and the like. It's always good to compile as many sources as possible - which might be at times contradictory - and at best to consult the original source. It's always quite important to ask yourself: who stands behind it? What authority, NCO, or other entity? There are NCOs that are funded because of conflicts, they don't have any interest that conflicts are over. And then you have to know: how do they work? Do they conduct regular monitoring, fieldwork, or other research activities? Who works there? Many of the NCOs also engage in migration policy and have different views than ours from the FOM. Always read the 'about us': how is the funding, who are the partners? If there is no 'about us', that's a bad sign, as is no 'contact' [information]. It's up to you to decide what sources have a higher value. That is to be justified in the decision*. Sure, you can't always do everything; you have a lot to do - so focus on the important information! (Fieldnotes, country analyst, basic training, autumn 2012)

Her advice appears revealing in at least two respects: on the one hand, compiling COI seems to depend crucially on trusted sources - original sources and sources that disclose their methods and positionality - and to address the agendas of other producers of information. On the other hand, a high 
degree of discretion seems to be involved in the selection and 'valuing' of sources. The country analyses I read were compiled from - and are supposed to balance, in the view of country analysts - various sources, including the media, government and NGO reports. They also drew on data gathered in so-called 'fact-finding missions' by country consultants (involving interviews and informal talks with various local actors). They also comprise often heavily cited COI reports produced in other countries in the EU (or in the US).

Take as an example a so-called COI-"note": this is a mid-range COI that is not case-specific, but considers a specific topic related to persecution, not the 'general situation' in a country (see Figure 7). It declares to provide answers to questions about the "situation of homosexuals in Uganda" - an issue of high topicality at the time of my research in the office. The note was addressing, according to the grey box on the front, three main questions: What Ugandan laws refer to homosexuality and what punishment do they potentially foresee for this? How are these laws applied in practice? Are there indications of persecution of homosexuals by the state or society in Uganda? The "contracting entity" for the report was indicated as the under the "Aegis [Federführung] Uganda" (see part on APPA in section 5.2.3). The sources cited in the note included, for instance, the Ugandan Penal Code Act, a Human Rights Watch "Update", a UK Border Agency COI Report, a Conversation with Representatives of the Ugandan Consular Agency, the BBC, and a Ugandan newspaper. After discussing the questions above in light of these sources over several pages, the country analyst provided a brief concluding "commentary" in which he provided his reading of the "situation of homosexuals" in Uganda at the time.

While such reports are usually classified as "internal", there seemed to be a tendency to extensively share $\mathrm{COI}$ information and reports on the EU level via the European Asylum Support Office (EASO). ${ }^{26}$ The Swiss COI note also featured a disclaimer at the very end that declared, "The note at hand was created by the country analysis according to the common EU guidelines for the processing of information about countries of origin" (COI note, fieldwork materials, 2013).

26 https://www.easo.europa.eu/(last accessed June 2, 2020) 
Figure 7: COI note concerning the "situation of homosexuals" in Uganda

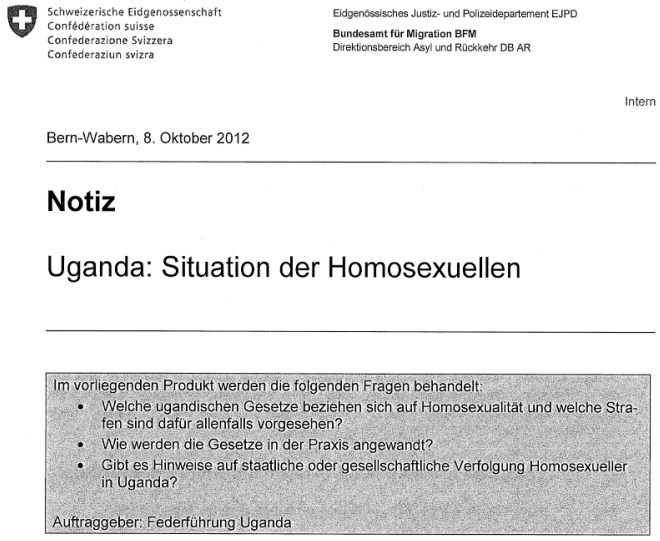

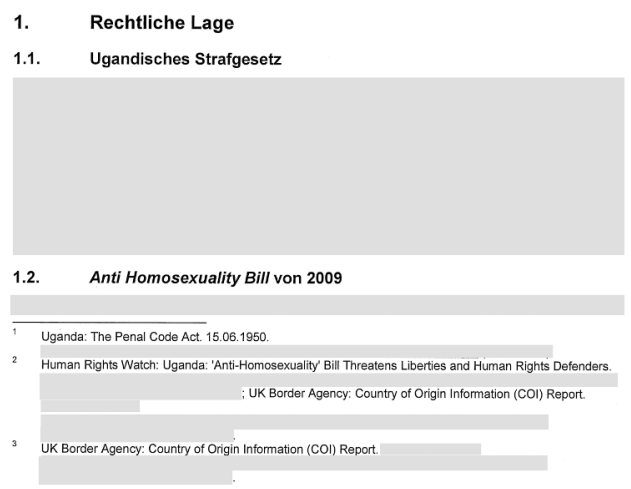

(Source: Fieldwork materials; 2013)

The "fact-finding missions" mentioned above are now often conducted in collaboration between country analysis services from different European countries as well. This indicates the increasingly significant networks in which the knowledge practices of COI have to be situated. However, the information generated in networks of immigration authorities does not necessarily remain in these. As Rosset and Liodden (2015) found in their analysis of a recent Danish COI report on Eritrea:

Even though conclusions may be heavily disputed, we observe the ways in which the Danish [Eritrea] report began to take on 'a life of its own' in the 
international sphere. Information that can legitimise restrictions seems to be picked up very quickly, especially by political actors with a populist and/or anti-immigrant agenda. (Rosset and Liodden 2015, 29)

While COI usually are inscribed in reports, they may also take the form of presentations. During my fieldwork, I was invited to, and attended, such presentations on several occasions. Furthermore, COI and country analysts are not the only sources of knowledge to associate narratives with the spatiotemporal 'realities' of persecution on the ground. Caseworkers may themselves have incorporated such 'knowledge': an academic specialisation such as Middle Eastern studies, or work experience in a country or region of origin - or they may rely on co-workers who are 'experts'. They may also consider interpreters' evaluation of dialects of claimants or their insider knowledge in the evaluation of purported flight motives. This means that interpreters are not only crucial mediators of the communication in asylum hearings (see Latour 2005, 135), but they are moreover important sources of knowledge as 'language or cultural experts'. Interpreters' subjective evaluation of an applicant, their comments or objections may in turn trigger further clarifications (see also Noll 2005; Scheffer 2001), such as linguistic tests. In practices of case-making, information about the countries of origin neatly blend with official COI, incorporated knowledge (from expert to hearsay), and what the interpreter says. Only in written accounts authored in decisions* does the citable 'information' of inscription devices of country analysts trump other forms of knowing: "states of conviction" (see Chapter 7) have to be turned into reasonable resolutions.

\section{Linguistic expertise}

For considering applicants' eligibility to asylum, identifying their nationality is essential. This poses huge difficulties since, as Torpey $(2001,269)$ notes, "a person's nationality simply cannot be determined without recourse to [passports and other identity] documents. As an ascribed status, it cannot be read off a person's appearance". In many cases, applicants do not provide such documents, which means that the identification of claimants in the asylum procedure poses considerable challenges. ${ }^{27}$

27 Papers are not provided for various reasons I cannot detail here. In the hearings, applicants often say that they lost their identity and travel documents on the route or did not 
In the absence of documents, caseworkers either draw on technologies of linguistic expertise (see Eades, 2009) or country of origin questions (see section 6.4.1) to associate applicants with a country of origin (which is equally the country to which they are potentially deported). For linguistic expertise, caseworkers in the asylum office can commission so-called "LINGUA tests" to inscribe the linguistic and cultural context of socialisation of claimants. In such tests, external experts interview claimants on the phone and evaluate their speech and knowledge. They ask questions about culture, local habits, prices of goods, places, schooling, or politics and analyse linguistic specificities of the interviewees' vocabulary and expressions. Then they write a confidential report for the migration office that contains meticulous descriptions of their analysis and an assessment of the likelihood of socialisation in the purported country of origin or an alternative. LINGUA tests are relatively costly and laborious but are favoured by many officials because they are considered more objective than non-expert questions in the hearings:

The LINCUA analysis is of course great, because you can, depending on the language, say really precisely where people come from: this means from which area, less from this and this city. Depending on the dialect they have, this is quite the safest possibility, because you cannot necessarily fake the dialect when speaking. (Interview with caseworker, autumn 2013)

LINGUA tests are thus quintessential inscription devices of asylum case-making: after a complicated and - for non-linguists - untraceable procedure, they provide caseworkers with a simple tick response to their question of origin. Figure 8 shows the example of not "definitely" but at least "most likely from: Sierra Leone".

take them from home. A common explanation by caseworkers for the largely absent papers is that applicants fear to be sent back (more easily or quickly) if they provide papers. 
Figure 8: LINGUA expertise form

Confidential - Not for editing

\section{Expert}

I have written the following expertise ${ }^{*}$ concerning the area of socialisation of the named person on request of the Federal Department of Justice and Police (FDJP) and through the scientific unit LINGUA. According to my working contract I am obliged to fulfil this assessment as an independent specialist, working in a confidential manner and not responding to any kind of directives. My work with LINGUA undergoes continuous quality controls.

\section{Expertise}

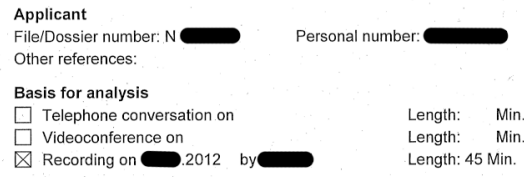

Areas of analysis

The determination of the area of socialisation has been based on

$\bigotimes$ analysis of the knowledge of the country and its culture.

This analysis examines important domains relative to the experience and the knowledge the applicant has of the geographical area and/or socio-ethnical background he claims to come from.

$\bowtie$ linguistic analysis.

This analysis examines the applicant's language(s)/dialect(s) in terms of phonology, morphology, vocabulary and other features.

$$
\text { Result }
$$

The analysis allows me to state that the predominant socialisation of the applicant took place within the following geographical area and/or socio-ethnical background:

$\square$ definitely:

$\square$ definitely not:

冈 most likely: Sierra Leone

$\square$ either: or

According to Swiss procedural administrative law (VWVG; SR 172.021) the expert's result equals an expertise. An expert is a person who, due to his/her specific knowledge, is directed by the competent The result will be put to protocol orally or be the subject of a written report (Art. 12 Bst. e VWVG).

(Source: Fieldwork materials, autumn 2013)

Such tests, however, are not obtainable for all countries and may not deliver clear results in every case. According to an internal instructor, they are only advisable in cases in which their outcomes are decisive for the case. But if used, they become crucial evidence - and arguments (see subchapter 6.5) - in support of or against an applicant's purported origin. They extend the scope of defining and inscribing 'origin' in effective, recordable ways. They authorise associations of applicants to the state they fled according to their performance of knowledge and speech linked with it as a space of socialisation. Of course, one could object that they have at least two major pitfalls. Firstly, spaces of linguistic and cultural socialisation rarely coincide with national territories, and these spaces are becoming increasingly hybrid (see Pieterse 1995). Secondly, they build on a notion of sedentarist socialisation which is not congruent with the migratory trajectories (Garelli and Tazzioli 2013,1014 ) and potentially multi-local livelihoods (see Thieme 2008) of many 
claimants. However, these 'problems' usually do not limit their effectiveness as inscription devices that provide crucial associations to resolve cases.

\subsubsection{Coordination Devices}

Technologies that allow for the coordination of practices of asylum case-making are a further crucial form to be analytically distinguished. I suggest considering technologies of coordination to make sense of how practices become orientated "toward some kind of good that delimits the reality taken into account" (Thévenot 2002, 75-76). I draw here on Thévenot (2002) who introduced the notion of coordinating devices to grasp complex pragmatic conventions. Conventions in his terms do not refer to "collective agreements", but to "nothing more than a limited agreement about selected features people use to control events and entities" (ibid., 83n18). They thus render different perspectives and approaches (see section 8.1.2) commensurable by generating a sort of minimal agreement about what exists, what is inconvenient, and what irrelevant for case-making - they are a form of heuristic that has materially stabilised in a device and transport particular classifications for practices of case-making. In my view, administrative guidelines and forms, but also digital interfaces, can be understood as such coordinating devices. They are all supposed to coordinate and align events and entities of case-making to some extent, but do not imply (nor allow for) complete alignment. Such devices thus coordinate case-making by formatting work in certain categories of governing asylum (see subchapter 8.2).

Forms should not only be considered as the ubiquitous inscription and writing devices they are, but also as quintessential coordinating devices of case-making. On the one hand, forms coordinate much of the interaction with asylum seekers who have to fill such forms to make themselves legible and/or their applications formally submitted (e.g. Gill 2014). On the other hand, forms are excessively used inside administrations to render the production of records both more efficient and considerations selective. I introduce two different types of forms in PART II: the first is a personal data form, filled out by those seeking asylum in reception centres, which coordinates the registration of applicants-with-cases (in subchapter 6.1; see also Gill 2014), the second a triage form filled out by caseworkers in the reception centres after the first hearing coordinating a case file's further trajectory 
through the processual events of case-making (see subchapter 6.3). ${ }^{28}$ In this section, I will instead concentrate on two other types of coordination devices crucial for case-making: asylum practice" guidelines, so-called APPA and digital and analogue databases.

\section{Asylum practice* and APPA}

The asylum practice" is - as the name indicates - a technology for coordinating practices of asylum case-making and thus stabilising the dispositif. As a senior suggested in the basic training:

At work: read Article 3 and keep the refugee convention in the back of your mind - however, it is not written there in black and white who counts as a refugee according to current case law. (Fieldnotes, senior's recommendation in basic training for new caseworkers, autumn 2012)

Who counts as a refugee and how certain types of cases are to be approached thus cannot be found in the law itself but are rather a matter of knowing the asylum practice*. Asylum practice" combines a discourse about how things are to be done for certain types of cases with codified, authoritative knowledge of internal guidelines. More specifically, asylum practice* becomes internalised through heuristics and exemplars (see sections 4.2.3-4), is written in internal guidelines, and conveyed, or performed, in institutional events such as recurring internal training sessions and information meetings. The latter are particularly important for making caseworkers and seniors aware of changes in the asylum practice* of a certain country or domain. Rare but crucial events are those concerned with defining the terms of changes in a practice" (see section 7.2.2 for an example of such an event).

The asylum practice* materialises in internal guidelines - or coordination devices - called APPA, ${ }^{29}$ an acronym composed of the German and

28 See also section 8.2.2 for an account of how case trajectories may be affected by a non-legal, calculative "kind of good that delimits the reality taken into account" (Thévenot 2002, 75-76).

29 APPAs for countries of origin are internally also referred to as the "country practice".*. This country practice of each country is under the aegis [Federführung] of a head of section and one or two caseworkers of this section. The documents outlining the practice - APPAs only exist, however, for countries of origin from where a significant share of asylum applicants emanates. The documents can be downloaded from the internal COI database 
French terms Asylpraxis and Pratique en matière d'asile, respectively. APPA is used simply as an abbreviation for asylum practice. APPA guidelines contain specific "guidelines for practice", which namely outline what persecution scenarios are well-known in a specific country of origin (similar to operational guidance notes (OGN) in the UK; see Gibb and Good 2013, 298-99). They also indicate potential obstacles to removal, possibilities for identification measures (e.g. LINGUA tests or document analyses) or crucial considerations to take when cases fall into a specific thematic domain (e.g. gender-related persecution). APPAs are crucial devices of coordinating case-making in legal-pragmatic terms, not least because of their authoritative character: the compliance of caseworkers with these APPAs is regularly evaluated. A caseworker emphasised this authoritative character when I asked him about 'different approaches' that caseworkers tend to develop in case-making:

Researcher: Do you realise, if you work together with other people, that everyone has a slightly different approach?

Caseworker: Yes, of course. But then, very much is prescribed by the country practice*. For instance, I can't just say I have the feeling a Bahai in Iran is not persecuted. Here the country practice* just tells me, no, he is persecuted. Then you only have to see whether that is credible that he is persecuted due to his religious affiliation or not. And when I say, I have the feeling that a Pentecostal in Eritrea does not have any problems, then it says in the APPA, sure, he has a problem (laughs). Then I cannot value that myself, I just have to look, is it credible or not, that is what I can still determine. But it's a lot that is predetermined in this respect, your leeway is not huge, right? Because you have... the people who determine the practice, these are like neuralgic points, which can dictate a lot of things.

(Interview with caseworker, autumn 2013)

Hence, APPAs partially align 'slightly different approaches' of case-making by 'evoking' certain constellations of persecution that caseworkers cannot simply ignore. A senior once formulated this more positively when I asked

KOMPASS. Additional to the country doctrines, thematic doctrines exist for important topics such as gender-related persecution, family reunification, or exclusion from asylum. These are assigned to Federführungen as well, where the heads of section in turn have the competence for suggesting changes in the doctrine. This has been read as a form of "bottom up" policy-making (see Miaz 2014). 
him about the role of APPA: he said they primarily provide clues to what you need to pay attention. For instance, he continued, the APPA of Iraq contained information about how the persecution of Yazidi had highly intensified in the last years and questions which had to be clarified in this regard.

Guidelines such as APPA thus format case-making by outlining 'real' scenarios, which have to be considered. They provide clues for asking the right questions in hearings and state possibilities for further clarifications. They work towards the establishment and maintenance of a practice* doctrine to facilitate that similar claims are evaluated alike. This is, besides the effort to compile and maintain them, why they are only considered worthwhile to establish for countries of origin such as Eritrea, Syria or Afghanistan, which a larger share of the workforce has to deal with. For these countries of origin, caseworkers could draw on an APPA that was regularly updated to the latest developments. At times, new APPAs had to be compiled, as for instance in the case of Syria: As the share of Syrian applications was very small when I started my fieldwork, no APPA existed. When applications from Syria started to rise slowly and then from mid-2013 onwards even drastically increase, a doctrine was established and codified in an APPA (see excursus).

\section{Excursus: APPA Syria}

During my last period of my fieldwork in the headquarters, I attended a meeting between the head and vice-head and two caseworkers of a section who had the joint lead [Federführung] regarding Syrian asylum practice" in the office. Syrian applications had constantly risen and about 2000 were pending at that time. Syrians had increasingly claimed persecution in relation to the military service (desertion or conscientious objection). The meeting was about a "practice decision" concerning these types of claims: how should they be treated, what needed to be considered, and what did that mean for their resolution? In line with my duties of data protection (see Chapter 3), I will - instead of stating what was decided - introduce some of the crucial questions that were addressed in the meeting:

Was the envisaged practice change in line with the practice* of other key European countries, to avoid a 'pull effect', but also not to stay 'too far 
behind'? $?^{30}$ [A table distributed amongst the participants outlined the practice* of such key countries.] What different persecution constellations could be sensibly differentiated? [A document outlined a possible solution.] This led to questions like: What people were concerned? Should a difference be made between deserters and conscientious objectors? Could the supposed differences in drafting practices based on religion or ethnicity justify a different treatment in practice? Should a temporal boundary for the evaluation of such cases be erected to distinguish between those who fled before the civil war began and those who fled during it (for whom consequences could be considered to be more severe)? What forms of evidence were relevant for persecution constellations? What were their legal basis and consequences (asylum, exclusion from asylum, temporary admission)? What distinctions were reasonable based on what was known about the draft practice of the regime? What distinctions could be practically achieved based on applicants' testimony and pieces of evidence? What standard of proof was to be applied in credibility assessments? What to do about central pieces of evidence that could be easily forged? (Field notes and documents, spring 2014)

The answers to these questions implicate what Thévenot $(2002,70)$ called "orders of worth" or pragmatic conventions of evaluating what shall count as persecution and what legal consequences this shall have.

The participants of the meeting discussed some of these questions intensely until they arrived at a conclusion. After the meeting, the head of the section had to defend their suggestion for the new practice* in a high-level meeting with the senior management of the office. After it was accepted there, the APPA was updated accordingly, and a scheme outlining the persecution scenarios and the crucial distinctions to be made was provided to the caseworkers by email and introduced at an internal meeting in the headquarters.

\section{Digital databases and interfaces}

Digital databases and interfaces are additional and increasingly significant types of coordination devices. Their scope goes far beyond simple digital storage and retrieval. They crucially format the coordination of practices

30 This indicates that identifying and incorporating 'best practice' examples strongly informs 'national' practices of governing asylum. 
of case-making by enabling certain forms of (super-)vision. Crucial for case-making is the central digital migration database called ZEMIS (Zentrales Migrationsinformationssystem). ${ }^{31}$ The senior official who introduced ZEMIS to new caseworkers and me declared it to be "one of the most important work tools" [Arbeitsmittel]. He said it serves to "map our work and provides the basics for [writing standard] letters, which we can extract from it" (Fieldnotes, basic training for new caseworkers, autumn 2012; see also subchapter 6.5). One could moreover find the procedural state of a case file in ZEMIS to "see what has happened until now" (ibid.). He mentioned that access rights to sections of the database vary and thus "we do not see everything". There are various other authorities - such as the cantons and the FAC - whose agents also have (partial) access. Importantly, he also highlighted that the management and controlling of asylum case-making is solely based on what is in ZEMIS: "ZEMIS is the basis of statistical analyses, controlling and strategic decisions in the asylum domain" (Handout, basic training for new caseworkers, autumn 2012). Thus, for the management, logging case-making in ZEMIS brings it into existence and makes it calculable and governable. By consequence, ZEMIS operates as the most crucial mediator between the centres of calculation (see section 8.2.1) and those doing casework.

How does data about case-making enter the database? While caseworkers in the reception centres have writing rights in ZEMIS's proceedings section, caseworkers in the headquarters do not. This is the task of a special unit in the migration office, the DSDE (Dienst für Sachdatenerfassung), which stands for "service for the registration of technical data". Caseworkers send them special forms for adding persons to a case file or registering the completion of a main hearing; asylum order templates already contain numeric codes on the last page indicating the transactions concluded by them. A copy of the last page of outgoing rulings is delivered to the DSDE for registration.

What vision of case-making does ZEMIS offer? It lists the asylum permit history which includes an overview of the distribution process, i.e., the allocation of the applicant to a canton. The most interesting part for case-making is the section about the state of the proceeding. Importantly, ZEMIS reveals the location of the physical case file, which can be a collaborator in any section of the SEM, the FAC, the archive, but also, for instance, the Federal Intelli-

31 While at the time of my fieldwork it did not yet contain actual records of cases, their incorporation as 'e-case files' [eDossier] was close to being launched. 
gence Service. If the case file is located in the asylum office, ZEMIS indicates the (acronym of the) official in charge of the proceeding. Here, a case file can be ordered or transferred to another official. "Over the years you'll know all the acronyms by heart" (Fieldnotes, basic training for new caseworkers, autumn 2012), the instructor said with a smile. The responsibility for correct registration lies with the person transferring a case file. Only the insertion of case files into (and removal from) the archive is automatically registered.

Altogether, a large and intricate assembly of different devices - software, hardware, delivery devices, forms and codes - and people populates the analogue-digital interface of the asylum dispositif. Its operation is fraught with peril, and a small defective update of software can lead to a breakdown of myriads of working processes, as I witnessed several times. For instance, all the affected members of the administration were repeatedly informed about breakdowns and malfunctions of the infrastructure per email. A search through my emails revealed that during one year of observation (autumn 2013 until summer 2014), IT infrastructure problems of varying severity and duration had occurred every few weeks. Both the Single-Sign-On (SSO) portal for accessing all databases (including ZEMIS) and ZEMIS itself broke down several times completely; moreover, on one occasion the electronic records and process management interface GEVER (elektronische Geschäftsverwaltung) and recording PT (Personal Time) were temporality interrupted, the new e-case files ${ }^{32}$ could not be accessed and all printers in the network went offline. A particularly persistent malfunction occurred at the interface connecting ZEMIS and the word-processing program, which is crucial for capturing data in all records to be written. It meant the applicant and editor data were merged inaccurately in the documents over more than four weeks. According to Latour (2005), it is exactly in such events of failure that the crucial mediating role of devices for practices becomes visible, which is otherwise taken for granted. On such occasions, processual events of case-making (see Chapter 6) were interrupted, suspended or became at least more complicated. And they frequently forced caseworkers to improvise (see Jeffrey 2013) and to find workarounds for the technological devices' failures.

32 As indicated above, e-files are supposed to replace analogue case files in the asylum office in the long run. At the time of research, they represented merely a sort of digital appendix to the analogue case files in the main procedure. In the test centre of the State Secretariat for Migration, however, e-files have already become the new standard. 
In sum, I have suggested here that databases and interfaces operate as crucial coordinating devices for asylum case-making. On the one hand, they format those who assemble cases, such as caseworkers, sections, divisions, or the whole office; and they capture output, working time, availability of caseworkers, and further data. On the other hand, they format what is assembled by listing case events and their outcomes, linking case networks, and registering case files' assignment for processing and their physical location. Overall, these devices stabilise a certain powerful vision of the assembler, the assembly and what is (re)assembled through the dispositif and are thus profoundly entangled in the ways in which asylum is governed.

Both types of coordinating devices introduced here - APPA and digital interfaces - assemble a particular perspective on asylum case-making. These perspectives imply certain values or "orders of worth" (Thévenot 2002) by coordinating what counts - as persecution (APPA) and as work (ZEMIS). Coordinating devices have thus a crucial impact on how cases are approached, not only by conveying a certain agreement about what 'counts', but equally "a common acceptance of what is ... irrelevant" (Thévenot 2002, 83n18).

\subsubsection{Writing Devices}

Technologies of writing are crucial for case-making as they are not only involved in the production of most records of cases, but allow for cases' resolution in written letters. ${ }^{33}$ I suggest that the associations to record asylum cases are to a considerable extent preassembled in writing devices such as forms, database queries, standard letters and boilerplates. I focus here on the latter two: Standard letters and boilerplates are key "writing devices" (Callon 2002) that are amongst the organisational "tools for managing complexity" (ibid.) and allow for (the illusion of) "collective writing".

More than four hundred standard letters populate the server in the SEM and they reflect the classification of legal avenues available for case-making

33 Darling (2014) provided a detailed account of how letters (from the Home Office in the UK) become crucial mediators of the relationships between the state and asylum claimants and create particular atmospheres and encounters in a drop-in centre for asylum seekers. In contrast, I consider letters here from the perspective of the sender, of those issuing standardised letters to give instructions to asylum applicants or convey decisions. 
(Bowker and Star 1999, 10-16). ${ }^{34}$ Standard letters are thematically organised in internally accessible folders labelled with a code number and a few keywords. The Microsoft Word program used has an interface to the ZEMIS central migration database installed, which allows caseworkers to automatically insert case-specific data (such as name, address and dates) into a standard letter and also add information about the caseworker editing it (such as identification codes, name and head of section).

If there is no standard letter for a certain purpose and caseworkers have to write their 'own' letter, they still format the letter to appear as though it were a standard letter. As a caseworker told me, for a case we were discussing, that he composed a letter for the DNA test of the Angolan family the day prior and that it had cost him the whole day. No standard letter for this existed, so he said he had to write something that looked like a standard letter. This example suggests that a standard letter with its particular format not only makes work more efficient, but is moreover a key tool for coordinating administrative production: writing in the name of the state seems both to require a certain aesthetic and a performance of impartiality in written representation. This is mediated through prefabricated documents and document parts - or alternatively through the imitation of their visual and linguistic appearance.

Boilerplates [Textbausteine] are prewritten paragraphs that can be inserted at the relevant position in standard letters and (if necessary) adapted to the case at hand. They arguably shape the 'realities' of how to argue in a decision*:

Interviewer: And for the argumentation [in a decision*], what possibilities do you have, what room for manoeuvre?

Caseworker: Hmmm. We have lots of text modules, that is, boilerplates already written, which are sometimes specified for countries or some kind of assertion or so. And then you have to adapt them of course. Or you sometimes have to subsume under them what was asserted to you [by the claimant]. Or if for instance some module on credibility says this and that assertion contradicts the common logic of action, then you yourself have to argue why this is the case. But like that you have a somewhat more concrete yardstick

34 Each letter exists in the three administrative languages of Cerman, French and Italian, which makes about 1300 standard letters altogether. 
to measure it [the assertion] against. And for the countries, you partly have even more concrete things. (...). That's quite convenient [laughs].

(Interview with caseworker, autumn 2013)

According to this quote, what caseworkers consider they can 'argue with' in asylum decisions" is crucially mediated by the "boilerplates already written". These are not only "quite convenient" for decision"-writing, but offer a yardstick for how to argue regarding certain assertions. The most widespread ones formulate an official reading of legal articles and provide framings to argue with them in an asylum decision* (see subchapter 6.5). Usually, such boilerplates alternate in legal syllogisms with case-specific readings thereof.

Standard letters and boilerplates in the database have to be constantly updated to changes in law and policy. In the basic training, new caseworkers were warned that outdated boilerplates exist, and were asked to always check their content for timeliness. But it turned out that not only outdated, but also 'wrong' standard letters and boilerplates exist. In a conversation, a caseworker recounted how she once probed the legal relation established by a standard letter that she wanted to use:

Caseworker: Often, they rejected applications for reconsideration [of asylum claims] and then charged a fee of six hundred Francs. And then, I just had a closer look at this. I looked into the law on administrative procedures and looked whether there is case law on this. Then I realised, when it comes to legal aid, Article 65, paragraph 1 on the relief of procedural costs and paragraph 2 on legal representation free of charge ... this is actually not possible: you can't say first, it [the application for reconsideration] does not have 'any prospect of success', enter into the substance of the case, and thereafter charge a fee - since if it had had 'no prospect of success', you would have not been allowed to enter into the substance of the case in the first place.

Researcher: Yes, this is contradictory [we both laugh].

Caseworker: Yes, but there is a standard letter, where it is written like that, then it must be possible; just because some 'fool' has once written this. This is damn great, you just have to enter the $\mathrm{N}$ number [of the file] and it writes the letter all by itself, you just have to adjust the argumentation a little.

Researcher: But how should you find out what applies? I guess for many people the understanding and interest in how things look behind the curtain lacks, doesn't it? 
Caseworker: Totally, if there is a STAD [abbreviation used for standard letters], then it has to exist. But now there exists a standard letter which is just wrong, obviously.

(Interview with caseworker, autumn 2013)

Such 'wrong' standard letters are certainly rare. But they reveal the potential influence of inconspicuous "writing devices" in co-producing realities and mediating practices of governing asylum (see Callon 2002), where a legally inexistent connection can become unquestioned standard practice. Such connections, in turn, entail reassembling applicants' accounts in terms that may or may not be contested later on (in an appeal, for instance) and capture them in a peculiar association with the state. Yet, as the example of standard letters shows, the re-cording of applicants' lives enacted in writing decisions" is partly black-boxed by such technological devices at work (see also Murakami Wood and Graham 2006, 186).

From a perspective of case-making, standard letters and boilerplates crucially mediate what legal pathways are perceived to exist and what consequences they have. For many constellations, they offer a shortcut of how a particular constellation is translatable into legal text and action. They provide a standard response to problems which caseworkers are expected to - and usually gladly - pursue. Boilerplates offer a tested textual bridge to associate the specific case to the general practice for all or certain types of decisions; standard letters and other templates are devices that enable caseworkers to act in a scripted manner and advance the case on its trajectory to resolution. At the same time, they document work as they return into the case files as testimonial records of these actions (Vismann 2011b, 102). In short, they are crucial, yet hardly acknowledged, mediators in the governing of asylum. As is the case with many technologies, their mediating role becomes particularly visible if they fail (Latour 2005, 39). And they are implied in an uneven 'account-ability' - an ability to account for - between those engaging in writing and those subjected to it:

We must not forget that collective action is always tyranny. It is a tyranny of the past acting on the present and the future and a tyranny of those who write acting on those who are permanently excluded from writing. This is the other side of the management of complexity: the domination of those who 
have access to the tools without which management would not be possible. (Callon 2002, 214)

This tyranny of collective action - the vastly uneven agentic formations of those equipped with devices for managing the complexity of asylum applications and those subjected to such devices' effects in asylum encounters and the records that are re-cording their lives - is also a crucial feature of governing asylum. 
